\title{
Foot Contact Estimation for Legged Robots in Rough Terrain
}

\section{Conference Paper}

Author(s):

Wagner, Lucas; Fankhauser, Péter; Bloesch, Michael; $\underline{\text { Hutter, Marco (D) }}$

Publication date:

2016

Permanent link:

https://doi.org/10.3929/ethz-a-010643823

Rights / license:

In Copyright - Non-Commercial Use Permitted

Originally published in:

https://doi.org/10.1142/9789813149137_0047 


\title{
Foot Contact Estimation for Legged Robots in Rough Terrain
}

\author{
L. Wagner*, P. Fankhauser, M. Bloesch and M. Hutter \\ Robotics Systems Lab, ETH Zurich, \\ Zurich, 8092, Switzerland, \\ * Email: wagnerl@student.ethz.ch \\ www.rsl.ethz.ch
}

\begin{abstract}
Accurate sensing of the terrain shape is a key issue for robots moving in rough environments. For legged robots, an important aspect is the terrain inclination for each foot which is in contact with the ground. Knowledge about the terrain inclination is required to design controllers that mitigate the risk of slippage by minimizing the tangential forces applied on the ground. In this paper, we introduce a method to estimate the contact surface normal for each foot of a legged robot relying solely on measurements from the joint torques and from a force sensor located at the foot. The force sensors at the foot optically determine the deformation of the foot to estimate the force applied to the sensor (optical force sensors). We formulate a measurement model of the optical force sensor and combine it with the joint torque measurements in an Extended Kalman Filter (EKF). The resulting method is able to determine the contact force and surface normal through contact of the foot with the ground. The approach is implemented and evaluated on a torque-controllable quadrupedal robot and shown to reliably estimate the surface normal during dynamic motions for each leg individually.
\end{abstract}

Keywords: Legged Robotics; Terrain Estimation; Surface Normal; Optical Force Sensing

\section{Introduction}

Legged robots are advantageous for overcoming rough and unstructured terrain because their locomotion is based on the support of discrete footholds. This gives legged robots high mobility and allows them to reach places otherwise inaccessible for tracked or wheeled vehicles. However, maintaining stability while moving is a key challenge for legged robots and significant research has gone into approaching this issue. One major difficulty when walking over rough terrain is avoiding slippage. To this end, controllers have been developed which minimize the tangential forces at the ground contact. $^{1-4}$ These methods require knowledge of the ground surface normal 
for each foothold supporting the robot.

Previous work on legged locomotion on inclined surfaces can be split into reactive and proactive approaches. ${ }^{5}$ Reactive control strategies do not require prior knowledge about their environment, however they are limited to relatively modest slopes. For a robot executing a statically stable walk where three feet are always in contact with the ground, this can be done by fitting a plane through the three contact points. ${ }^{6}$ Estimating the ground normal during dynamic walking with only two feet in contact with the ground was presented using the history of the footholds. ${ }^{4}$ Proactive control strategies have been shown to work on much more challenging terrain, however they require prior knowledge of the terrain. Some assume perfect knowledge of their environment and can plan the footholds accordingly ${ }^{2}$ while others use range sensors to reconstruct the terrain online. ${ }^{7}$

In this work, we introduce a novel method to estimate the ground surface normal at the foot contact point for each foot individually. Our approach does not require prior knowledge of the environment (reactive approach), but significantly extends over existing plane fitting methods. The presented method relies on redundant measurements from torque measurements at the joints and measurements from an optical force sensor at the foot-tip, to estimate the direction of the surface normal for each ground contact. This method allows us to use proprioceptive measurements to estimate the ground normal without the use of any additional sensors.

In the following, we give an overview of our setup and introduce the optical force sensor and its measurement model (section 2). In section 3, an intensity measurement model and its parameter identification is described. Section 4 presents an estimator to fuse the measurements from the force sensor with the measurements from the joint torques. We illustrate our experiments on a quadrupedal robot in section 5 and discuss our approach and future work in section 6.

\section{System Overview}

\subsection{Torque Controlled Legged Robot}

The method presented in this paper was developed for torque controlled legged robots without any restriction on the number of legs or the leg configuration, as the ground normal is estimated independently for each foot. The main requirement is that an estimate of the robot's pose and ground contact force is available. For fully-actuated torque controlled robots the contact force can be estimated by leveraging the equation of motion together with joint torque measurements. 


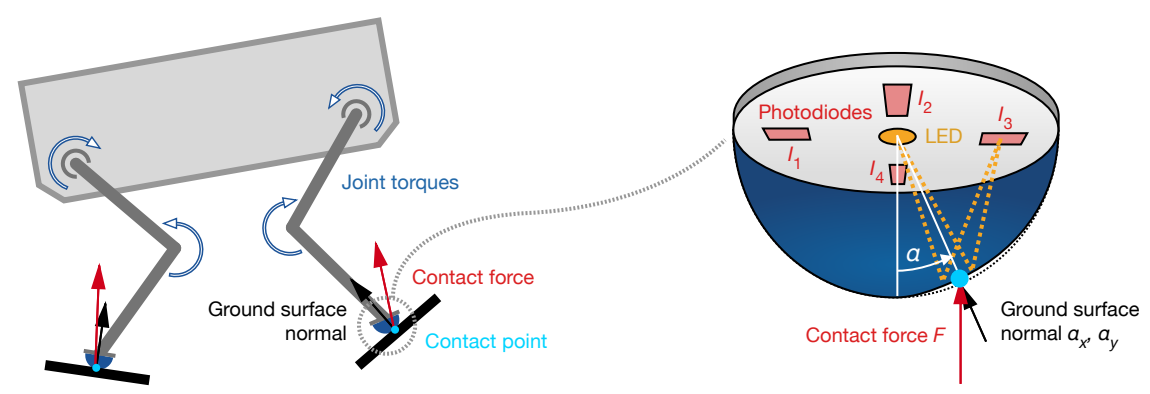

Fig. 1. The ground contact with the slope leads to a deformation of the elastic hemispherical dome of the optical force sensor at the contact point. The contact point is parametrized with the angles $\alpha_{x}$ and $\alpha_{y}$.

\subsection{Optical Force Sensor}

Optical force sensors use the intensity of refracted light to measure the deformation of their elastic surface. ${ }^{8,9}$ The sensor used in this work (OptoForce 3-Axis Force Sensor 50mm) ${ }^{10}$ consists of an infrared LED and four photodiodes inside a hollow elastic hemispherical dome (see figure 1). The photodiodes measure the infrared light reflected off the walls of the dome. The light intensity captured by each photodiode is approximately proportional to the distance of the wall to the sensor. When a force $\vec{F}$ is applied on the elastic dome, the walls are deformed and the light intensity captured by the photodiodes changes accordingly. The forces in $x$ - and $y$-direction can be obtained from the difference in intensity between the two opposing photodiodes for each direction: $F_{x / y}=\left(I_{1 / 2}-I_{3 / 4}\right) / 2$. The force in $z$-direction is given by the average of the four measurements: $F_{z}=\left(\sum I_{i}\right) / 4{ }^{8,10} \mathrm{~A}$ scaling factor is then applied by the manufacturer to all forces to get the calibrated force. The manufacturer-supplied implementation assumes that the force is applied from the top. In this paper however the four temperature compensated intensity measurements are used, which increases the measurement dimension from $\mathbb{R}^{3}$ to $\mathbb{R}^{4}$.

In this work, we assume that there is one contact point with the ground for each foot and that the contact point lies on a sphere of constant diameter (the deformation is negligibly small). With these assumptions, the contact point can be fully described using two coordinates. Because the foot surface is assumed spherical, the ground surface is always a tangential plane at the contact point. The contact point is described using two angles $\alpha_{x}$ and $\alpha_{y}$, which represent the inclination of the ground plane in $x$ - and $y$-direction. 


\section{Modeling of the Optical Force Sensor}

\subsection{Intensity Measurement Model}

For forces with arbitrary point of application, there is no simple physical model for the intensity measurements $\tilde{I}$ of the optical force sensor. To this end, we derive a heuristic model and fit it to data obtained from the sensor together with ground truth measurements.

We have tested multiple candidate models and compared their root mean square error compared to the ground truth measurements. The models we used were of the form $\tilde{I}=S(\vec{\alpha}, \Theta) \vec{F}$, where the sensitivity $S(\vec{\alpha}, \Theta)$ is a function of the contact angles $\vec{\alpha}$. The best results were obtained with the following model for the intensity $I$ of one photodiode:

$$
I=\mathcal{M}(\vec{F}, \vec{\alpha}, \Theta)=\left[\begin{array}{ccccc}
1 & \alpha_{x} & \alpha_{x}^{2} & \alpha_{y} & \alpha_{y}^{2}
\end{array}\right] \Theta \vec{F}+\text { bias }
$$

where $\Theta \in \mathbb{R}^{5 \times 3}$ is the parameter matrix which has to be identified, $\vec{F}$ is the actual force applied to the sensor and $\alpha_{x}, \alpha_{y}$ are the coordinates of the ground contact point. The same model is applied to all four photodiodes, with different parameter matrix for each photodiode (yielding 60 parameters in total).

The model assumes that the intensity is dependent on a linear combination of the three forces with a sensitivity that is a second order polynomial of the angles $\alpha_{x}$ and $\alpha_{y}$. The angle terms are required to capture the changing sensitivity dependent on the contact point.

\subsection{Parameter Identification}

To estimate the model parameters a Maximum Likelihood Estimator (MLE) was used together with the following assumptions:

(1) The four intensity measurements are conditionally independent on the force and the contact point.

(2) There is a additive Gaussian noise on the measurement model:

$$
\tilde{I}=\mathcal{M}(\vec{F}, \vec{\alpha}, \Theta)+\varepsilon, \quad \varepsilon \sim \mathcal{N}\left(0, \sigma_{I}^{2}\right) .
$$

With these assumptions, the model parameters can be evaluated individually using the MLE. The parameters to be identified are the model parameters $\Theta$ and the variance $\sigma_{I}^{2}$. This results in an estimator of the form:

$$
\Theta_{\mathrm{MLE}}, \sigma_{I, \mathrm{MLE}}^{2}=\arg \max _{\Theta, \sigma_{I}^{2}} p_{\mathcal{M}}\left(\tilde{I} \mid \vec{F}, \vec{\alpha}, \Theta, \sigma_{I}^{2}\right)
$$


where the probability is

$$
p_{\mathcal{M}}\left(\tilde{I} \mid \vec{F}, \vec{\alpha}, \Theta, \sigma_{I}^{2}\right)=p_{\mathcal{M}}\left(\varepsilon=\tilde{I}-\mathcal{M}(\vec{F}, \vec{\alpha}, \Theta) \mid \vec{F}, \vec{\alpha}, \Theta, \sigma_{I}^{2}\right) \sim \mathcal{N}\left(0, \sigma_{I}^{2}\right) .
$$

The parameters $\Theta_{\mathrm{MLE}}$ and $\sigma_{I, \mathrm{MLE}}^{2}$ were found by minimizing the negative log-likelihood function $-\ln \left(p_{\mathcal{M}}\left(\tilde{I} \mid \vec{F}, \vec{\alpha}, \Theta, \sigma_{I}^{2}\right)\right)$.

\section{Contact Estimation}

We design an Extended Kalman Filter (EKF) to fuse the measurements from the optical force sensor and the joint torque sensors for each foot/leg. The EKF allows us to estimate and filter the measurements as a timeseries. By combining these sensor signals, we can achieve

a) a more accurate and reliable estimation (including uncertainties) of the contact force $\vec{F}$ than it would be possible with only one of the sensor modalities,

b) an estimation (including uncertainties) of the point of contact (equivalent to the surface normal), which would not be possible with only one of the sensor modalities.

The EKF states are the force at the contact point $\vec{F}$ and the angles of the contact point $\alpha_{x}$ and $\alpha_{y}$. The measurements are the force estimated from the joint torques $\tau$ and the intensity measurements $\tilde{I}$ from the optical force sensor. The state prediction model assumes that the states remain constant and any change is driven by noise. This noise incorporates the foot movement and the change in force as well as the actual noise. The measurement for the intensity is given by the model presented in section 3.1 and the force estimated through the measured joint torques is assumed to be normally distributed around the actual force at the contact point.

\section{Results}

We have evaluated our method on the torque-controllable quadruped robot StarlETH. ${ }^{11}$ The robot is equipped with torque and position sensors in all joints and an optical force sensor manufactured by OptoForce at the foottip. ${ }^{10}$ StarlETH uses an IMU and the joint positions to estimate its pose and velocity. ${ }^{12}$

The model parameters were estimated for the model introduced in sec. 3.1 using the MLE from sec. 3.2. For this an ATI mini45 force sensor was used as a reference sensor and placed on the horizontal ground underneath one foot. The contact position was changed by moving the robots body 

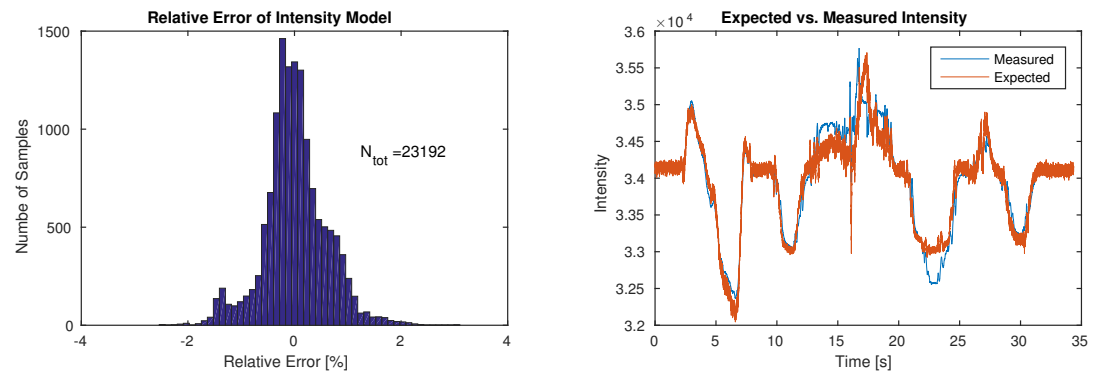

Fig. 2. The left figure shows the relative errors of the intensity model $\mathcal{M}(\vec{F}, \vec{\alpha}, \Theta)$ with regard to the actual measured intensity. The right figure shows the modeled intensity and the measured intensity over time.
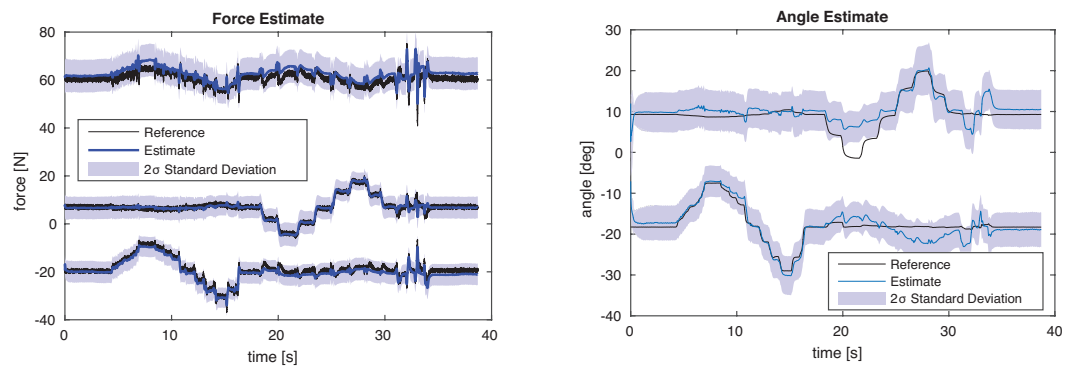

Fig. 3. The left figure shows the force estimate vs. the measured force with the reference sensor. The right figure shows the estimated angle of the contact point vs. the actual contact point.

around while keeping the legs on the ground. The contact position can then be deduced from the pose and the joint positions. The resulting model can accurately track the measured intensity as shown in fig 2 . The relative errors have a root mean square (RMS) of $0.59 \%$ for the depicted new measurement.

To test the performance of the estimator for the force and angle measurement, StarlETH was again placed on the ATI mini45 force sensor as described above and the EKF described in sec. 3.1 was run. This leads to a measurement were only the body is moved and the feet never leave the ground. Figure 3 shows that the force and angle estimates accurately track the reference signal. The main contribution to the force estimate comes from the torque measurements, but the additional information from the intensity measurements could help in situations close to joint singularities.

To verify estimator's performance while walking, the robot was placed on flat ground and then commanded to walk up a slope of known inclination. As the ground is assumed to be tangential to the foot, the ground normal is equal to the vector going from the contact position to the center 

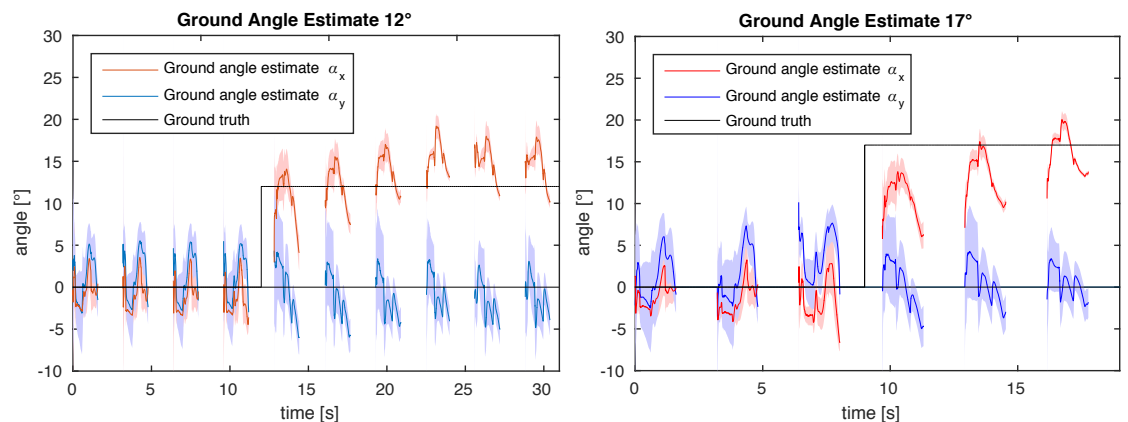

Fig. 4. Inclination angle estimates for walking onto a slope of $12^{\circ}$ and $17^{\circ}$ respectively. The shaded areas around the two signals represent the $2 \sigma$ confidence bounds. The signals in $\mathrm{x}$-direction change from $0^{\circ}$ to the respective angle when the foot steps onto the slope, which is inclined around the $\mathrm{x}$-axis. Meanwhile the estimate in $\mathrm{y}$-direction stays at $0^{\circ}$.

of the sensor. The robot first did a couple of steps on the horizontal ground before walking up the slope. Figure 4 shows the estimated ground inclination angles for a slope of $12^{\circ}$ and $17^{\circ}$ respectively. The RMS error of the estimation for the $12^{\circ}$ slope is $2.90^{\circ}$ in $\mathrm{x}$ - and $2.50^{\circ}$ in $\mathrm{y}$-direction. For the $17^{\circ}$ slope the RMS error is $3.35^{\circ}$ and $2.75^{\circ}$ respectively. The large errors at the beginning and end of each step are presumably due to an unfavorable leg position where the leg is extended, which leads to larger errors in the joint torque estimation.

\section{Conclusion and Future Work}

In this paper a method for estimating the ground contact forces as well as the ground normal using redundant force and torque measurements was presented. Comparing the results with a the reference force sensor shows that the method is capable of accurately estimating the foot contact force. The results of the walking experiments show that the method can be used to estimate the ground surface normal during walking. The errors at the beginning and end of each step are presumably due to the smaller force applied, which makes it harder for the algorithm to track the position as the difference between the intensities of the four photodiodes becomes smaller.

The results have shown that using information from optical foot sensors to estimate the ground surface normal is satisfactory, however improvements are still possible. Further work will consider projecting the joint torque measurement noise to the foot contact force estimation to improve the performance of the filter for unfavorable joint positions such as when the leg is fully extended. Further improvements are expected by adapting the EKF prediction step in order to integrate incremental joint position updates and thereby generating a better prediction. We also intend to use 
the ground normal obtained from this method in StarlETH's locomotion controller to improve its performance.

This work was supported in part by the Swiss National Science Foundation (SNF) through project 200021_149427/1 and the National Centre of Competence in Research Robotics.

\section{References}

1. M. Hutter, M. Hoepflinger, C. D. Remy and R. Siegwart, Hybrid operational space control for compliant legged systems, in Robotics: Science and Systems (RSS), 2012.

2. L. Righetti, J. Buchli, M. Mistry and S. Schaal, Control of legged robots with optimal distribution of contact forces, in IEEE-RAS International Conference on Humanoid Robots (Humanoids), 2011.

3. M. Focchi, A. del Prete, I. Havoutis, R. Featherstone, D. G. Caldwell and C. Semini, Ground reaction forces control for torque-controlled quadruped robots, in workshop on Whole Body Control at ICRA, 2014.

4. C. Gehring, C. D. Bellicoso, S. Coros, M. Bloesch, P. Fankhauser, M. Hutter and R. Siegwart, Dynamic Trotting on Slopes for Quadrupedal Robots, in IEEE/RSJ International Conference on Intelligent Robots and Systems (IROS), 2015.

5. J. P. Müller, in Intelligent Agent Systems Theoretical and Practical Issues, eds. L. Cavedon, A. Rao and W. Wobcke (Springer-Verlag, 1997), ch. Control architectures for autonomous and interacting agents: A survey.

6. A. Roennau, G. Heppner, M. Nowicki, J. Zoellner and R. Dillmann, Reactive posture behaviors for stable legged locomotion over steep inclines and large obstacles, in IEEE International Conference on Intelligent Robots and Systems (IROS), 2014.

7. P. Fankhauser, M. Bloesch, C. Gehring, M. Hutter and R. Siegwart, RobotCentric Elevation Mapping with Uncertainty Estimates, in International Conference on Climbing and Walking Robots (CLAWAR), 2014.

8. A. Tar and G. Cserey, Development of a low cost 3d optical compliant tactile force sensor, in IEEE/ASME International Conference on Advanced Intelligent Mechatronics (AIM), 2011.

9. C. Melchiorri, L. Moriello, G. Palli and U. Scarcia, A new force/torque sensor for robotic applications based on optoelectronic components, in IEEE International Conference on Robotics and Automation (ICRA), 2014.

10. OptoForce, Optical Force Sensors - Introduction To The Technology (2015).

11. M. Hutter, C. Gehring, M. Bloesch, M. H. Hoepflinger, C. D. Remy and R. Siegwart, StarlETH: a Compliant Quadrupedal Robot for Fast, Efficient, and Versatile Locomotion, in International Conference on Climbing and Walking Robots (CLAWAR), 2012.

12. M. Bloesch, M. Hutter, M. A. Hoepflinger, S. Leutenegger, C. Gehring, C. D. Remy and R. Siegwart, State Estimation for Legged Robots - Consistent Fusion of Leg Kinematics and IMU, in Robotics: Science and Systems (RSS), 2012 . 